\title{
Action of Gaseous Nitric Oxide on Some Physical and Chemical Parameters of Human Blood Samples
}

\author{
Andrew K. Martusevich1, Anna G. Soloveva1, Sergey P. Peretyagin'1, Anatoly F. Vanin'2 \\ ${ }^{1}$ Nizhny Novgorod Research Institute of Traumatology and Orthopaedics, Nizhny Novgorod, Russia \\ ${ }^{2}$ N.N. Semenov Institute of Chemical Physics of Russian Academy of Science, Moscow, Russia \\ Email: cryst-mart@yandex.ru
}

Received 10 May 2014; revised 23 June 2014; accepted 4 July 2014

Copyright (C) 2014 by authors and Scientific Research Publishing Inc.

This work is licensed under the Creative Commons Attribution International License (CC BY).

http://creativecommons.org/licenses/by/4.0/

(c) (i) Open Access

\section{Abstract}

We studied the metabolic changes induced by gaseous nitric oxide in whole blood samples in vitro. Blood samples were collected from healthy donors (Nizhny Novgorod station of blood transfusion). We carried out the direct bubbling of blood samples $(n=14)$ with gaseous flow with NO in a special appliance. We modeled standard conditions using the apparatus "Plazon" (concentration NO $800 \mathrm{mcg} / \mathrm{l}$ ). Middle power of gas flow was used. The blood sparging time was 2 min, and exposition time lasted $3 \mathrm{~min}$. Every blood sample volume was $5 \mathrm{ml}$. All the parameters were controlled before and after blood processing with NO. We tested lactate dehydrogenase activity in direct and reverse reactions spectrometrically by G. A. Kochetov's method. Aldehyde dehydrogenase activity was examined by B. M. Kershnhots's and E. V. Serkina's methods, superoxide dismutase-by T. V. Sirota's technology. Total protein level was examined by modified Louri's method. The concentration of lactate was tested with the automatic analyzer "SuperGL Ambulance". The indices of acidbase balance and blood gases partial pressure were estimated with special analyzer "ABL-77". Additional control of energy metabolism changes was accomplished with derivative parameters, such as coefficient of energy reaction balance and coefficient of substrate provision. Different changes of blood physical and chemical parameters are induced by NO-processing which was fixed in our experiments. There is an inhibition of erythrocytes energy metabolism, decreasing of plasma antioxidant reserves, moderate ionic disorders and of acid-base misbalance in blood samples in vitro. Besides, according to the indirect signs, the used regimen of NO-processing mainly affected erythrocytes, and stipulated methemoglobin formation. These data testify that the used dose of gaseous nitric oxide is too high for investigated human blood. In our opinion, registered negative effects of free NO may be eliminated by bound nitric oxide use (first of all in its natural form-dinitrosyliron complexes). 


\section{Keywords}

\section{Nitric Oxide, Blood, Lactate, Lactate Dehydrogenase, Superoxide Dismutase, Acid-Base Balance}

\section{Introduction}

At present many scientists, including physiologists, doctors etc., pay their considerable attention to the investigation of the efficiency of nitric oxide use in different areas of biology and medicine [1]-[5]. One of the variants of practical realization of NO-dependent technologies is generator of cold plasma with nitric oxide ("Plazon" apparatus) [6]. Although there are many papers about its clinical effects, but its molecular and cellular action of this form of free (gaseous) NO are low investigated [5]-[7].

There are two main components of Plazon-therapy: gaseous NO and cold plasma, that carried it. At modern scientific data, last substance is not so inert for biological objects. Cold plasma processing leads to microorganism elimination from different surfaces [8]-[10], surgical instruments [11], and even paper envelopes [12]. On the other side, using cold plasma may be a supplementary method for sanogenic effect to wound healing [10] [13] [14].

As for second component of the medical processing nitric oxide has a many-sided biological effect being also a molecular bioregulator in all the functional systems (vessels tone regulation [1] [5], neurotransmission [2], platelets aggregation [15]-[18], apoptosis [19] etc.). Now medical use of free NO is covered by newborn and adult respiratory distress syndrome [20] and primary hypertension [21].

Negative effects of NO are also shown. It is realized through peroxynitrite synthesis [1] [5] [22], which can produce the nitrosative stress [22]-[24]. In this connection estimation of physiological NO level in biological systems is a principal aim. During the last decades many investigations are dedicated to its direct or indirect scrutiny, but this task hasn't been determined yet till now. Therefore, the problem of exogeneous correction of NO level in biological tissues and substrates is being discussed [25]. Dose-dependent effects of NO are low examined too. In addition, there are singular data about action of NO on energy metabolism of different cells.

That is why the aim of this work is complex estimation of nitric oxide action on whole blood of healthy people.

\section{Materials and Methods}

\subsection{Material}

We tested reaction of the whole human blood $(n=14)$ on processing with NO-contained cold plasma. Blood samples were collected from healthy donors (Nizhny Novgorod station of blood transfusion).

All donors (from 20 to 40 years old) were physically and laboratory examined before blood delivery and had no any chronic or acute pathology. They are not smokers or alcohol abusers.

Protocol for this research project has been approved by Local Ethics Committee of the Nizhny Novgorod Research Institute of Traumatology and Orthopaedics.

\subsection{NO Generation Protocol}

We executed direct sparging of blood samples by gaseous flow with NO in special applicance for 2 min. NOcontaining cold plasma was generated with the apparatus "Plazon" (Russia). We modeled standard conditions of using the apparatus (concentration NO $800 \mathrm{mcg} / \mathrm{l}$ ). Middle power of gas flow was used. Blood sparging time was $2 \mathrm{~min}$, exposition time-3 min. Sparging was performed in glass vial. Every blood sample volume was $5 \mathrm{ml}$. Experiment was repeated tenfold.

\subsection{Methods of Investigation}

All the investigated parameters were controlled before and after blood processing with NO. We tested lactate dehydrogenase activity in direct and reverse reactions spectrometrically by G. A. Kochetov's method (1980). Aldehyde dehydrogenase activity was estimated by B. M. Kershenhots's and E. V. Serkina's methods (1999), 
superoxide dismutase—by T. V. Sirota's technology (1999). Total protein level was examined by modified Louri's method.

Lactate level was tested with the automatic analyzer "SuperGL Ambulance". The indices of acid-base balance and blood gases partial pressure were estimated with special analyzer "ABL-77".

For integral assessment of energy metabolism changes a number of derivative parameters (a coefficients of energy reaction balance [ERB] and substrate provision [SP]) were used. They are calculated with special formulas [26]:

$$
E R B=\left(L D G \operatorname{dir}^{2}\right) /\left(\text { LDGrev }^{2}\right) \times 100
$$

(LDGdir-activity of lactate dehydrogenase in direct reaction; LDGrev—activity of lactate dehydrogenase in reverse reaction).

$$
\text { SP }=\text { C }(\text { Lactate }) \times \text { LDGdir } / \text { LDGrev }
$$

(C(Lactate) - erythrocytes lactate level; LDGdir-activity of lactate dehydrogenase in direct reaction; LDGrev—activity of lactate dehydrogenase in reverse reaction).

\subsection{Statistical Analysis}

Statistical analysis of the data was performed with Statistica 6.0 program. Data were expressed as means \pm SE, the Student's t-test was used for detection of statistical difference.

\section{Results and Discussion}

Our experiments showed, that blood processing by gaseous flow with NO, generating with "Plazon" apparatus, clearly changed level of investigated parameters. Particularly, tested agent led to transformation of some enzymes functioning (Figure 1). So, lactate dehydrogenase activity in direct reaction was decreased (at 15\%; p < 0.05 ) with its elevation in reverse reaction (at $86 \%$; $<0.05$ ). This tendency indicates the reduction of erythrocytes energy reserves under blood NO-processing.

On our opinion, NO-induced decreasing of superoxide dismutase (at 39\%; $<<0.05$ ) has a compensatory value. It is known, that this enzyme is one of the main component of blood antioxidant system. Some investigators have demonstrates, that an action of cold plasma and NO on biological fluids associated with hyperproduction of free radicals [5] [10] [22]-[24]. In connection with it, the observed inhibition of superoxide dismutase activity (at $64 \%$; $\mathrm{p}$ 0.05) can be induced by its role in the bioradicals elimination.

We also studied, the whole blood sparging with NO-contained gas flow is not changed aldehyde dehydrogenase activity.

There are established NO-dependent metabolic transformations, first of all—disorders of lactate dehydrogenase functioning regimen with hyper activation of its reverse reaction, cause differently directed dynamics of lactate concentration in blood plasma and erythrocytes (Figure 2). For example, in blood plasma we observed minimal lowering of this metabolite level (at 6\%), while in erythrocytes this parameter was elevated at $11 \%$ ( $\mathrm{p}$ 0.05). We supposed these revealed changes of the lactate dehydrogenase activity and its substrate level can be considered as hypoxia signs, induced by action of too high concentrations of NO on human blood samples.

For complex estimation of erythrocytes energy metabolism we used two derivative coefficients, including lactate dehydrogenase activity in direct and reverse reactions and lactate level in these blood cells. It was stated, that NO-processing of biological fluid lead to decreasing both estimated coefficients (Figure 3). We fixed, that the coefficient of energy reactions balance (ERB) was reduced at 3.5 times concerning the control level $(\mathrm{p}<$ 0.05 ), and the coefficient of substrate provision was lowered at 1.6 times to the control one $(\mathrm{p}<0.05)$. These changes indicate clear energy deficiency, forming in the blood by NO-containing gas flow. Considering that erythrocyte level of lactate was moderately increased, principal mechanism of observed hypoxia associated with disorders of lactate dehydrogenase reactions balance. The present hypothesis is fully confirmed by more marked elevation of the ERB coefficient as compared with a coefficient of substrate provision.

We also tested other parameters, which illustrated cellular hypoxic state indirectly, at blood processing with nitric oxide. They are partial pressure of oxygen and carbon dioxide (Figure 4). A priori investigated changes of lactate dehydrogenase acitivity and blood lactate level should be associated with lowering of oxygen partial pressure in this biological fluid, but we registered the opposite tendency. This parameter elevated at $107 \%$ to control level $(\mathrm{p}<0.05)$. On our opinion, revealed experimental data were stipulated by specialties of nitric oxide 


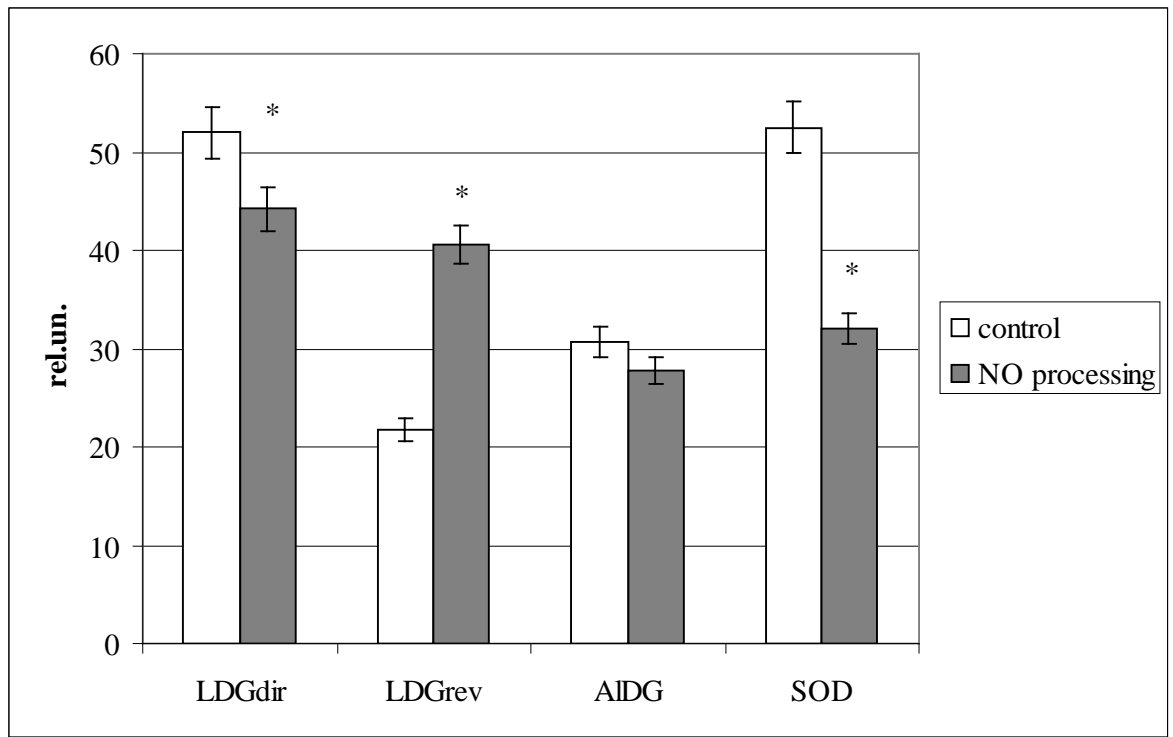

Figure 1. Blood enzymes activity at gaseous nitric oxide processing in vitro (LDGdir-direct reaction of lactate dehydrogenase; LDGrev-reverse reaction of lactate dehydrogenase; AlDG —aldehyde dehydrogenase, SOD—superoxide dysmutase; *Significantly different as compared to control level ( $\mathrm{p}<0.05$ ); rel. un.- nmol NADH/min·mg of protein).

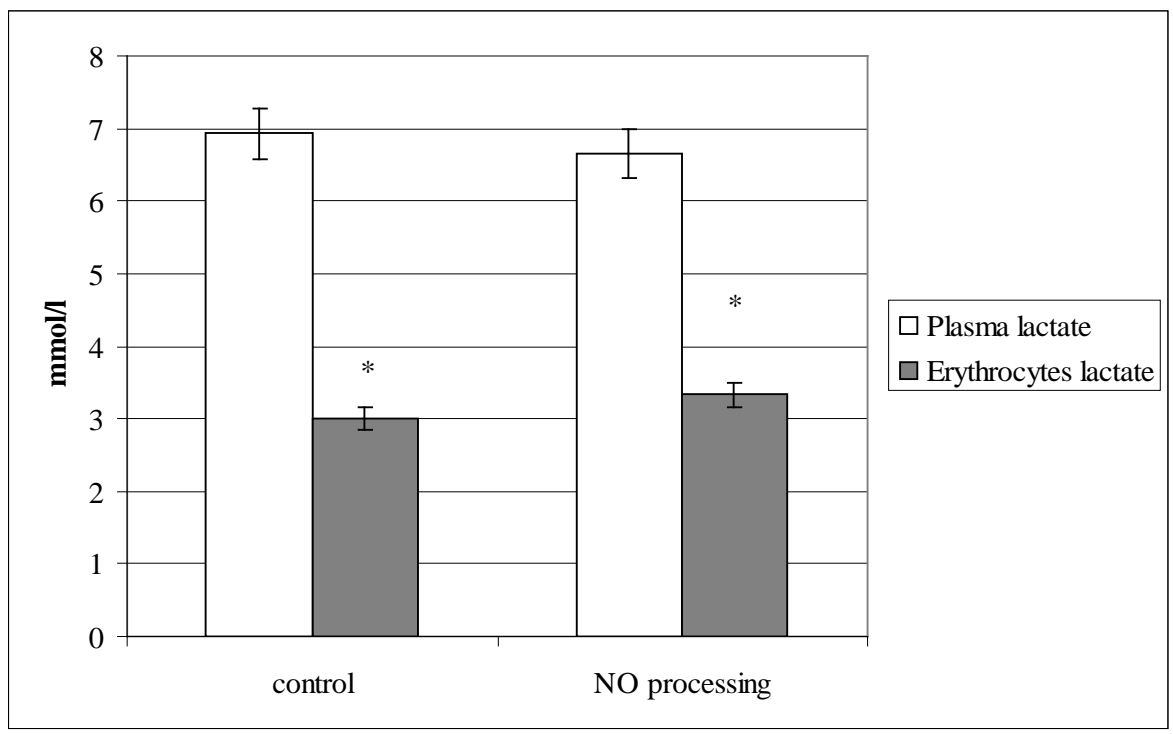

Figure 2. Plasma and erythrocyte level of lactate before and after NO processing of blood.

carrier-cold plasma, which contains significant amount of molecular oxygen. In plasma aggregate state this substances may be in metastable status [27] [28]. In addition, monoatomic oxygen may be presented in used gas flow [29]. Dissolution of these substances can produce a registered effect of hyperoxigenation with decreasing of partial pressure of carbon dioxide in NO-processed human blood.

We also stated, that biological fluid sparging with NO-contained cold plasma led to minimal acidification of blood samples ( $\Delta \mathrm{pH}=-0.08$ ), lowering of plasma bicarbonates level (at $44 \%$ to control; $\mathrm{p}<0.05$ ) and elevation of potassium concentration $\left(\Delta \mathrm{K}^{+}=0.43 \mathrm{mmol} / \mathrm{l}\right)$.

It's important to emphasize, at blood processing with gaseous NO we observed a "darkening" effect in biological fluids after sparging. On our opinion, it associated with methemoglobin formation under NO action, which was experimentally demonstrated by the same investigators [30]. 


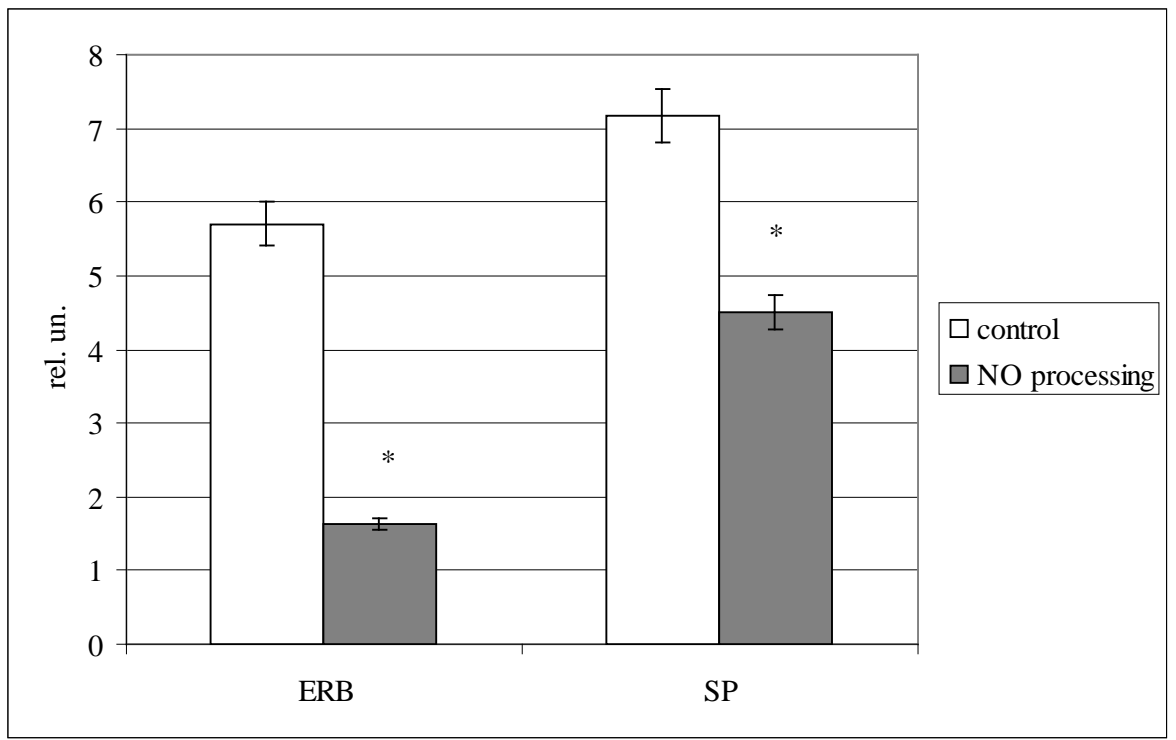

Figure 3. Plasma and erythrocyte level of lactate before and after NO processing of blood (coefficients of energy reactions balance [ERB] and substrate provision [SP] was calculated in relative units [rel. un.]).

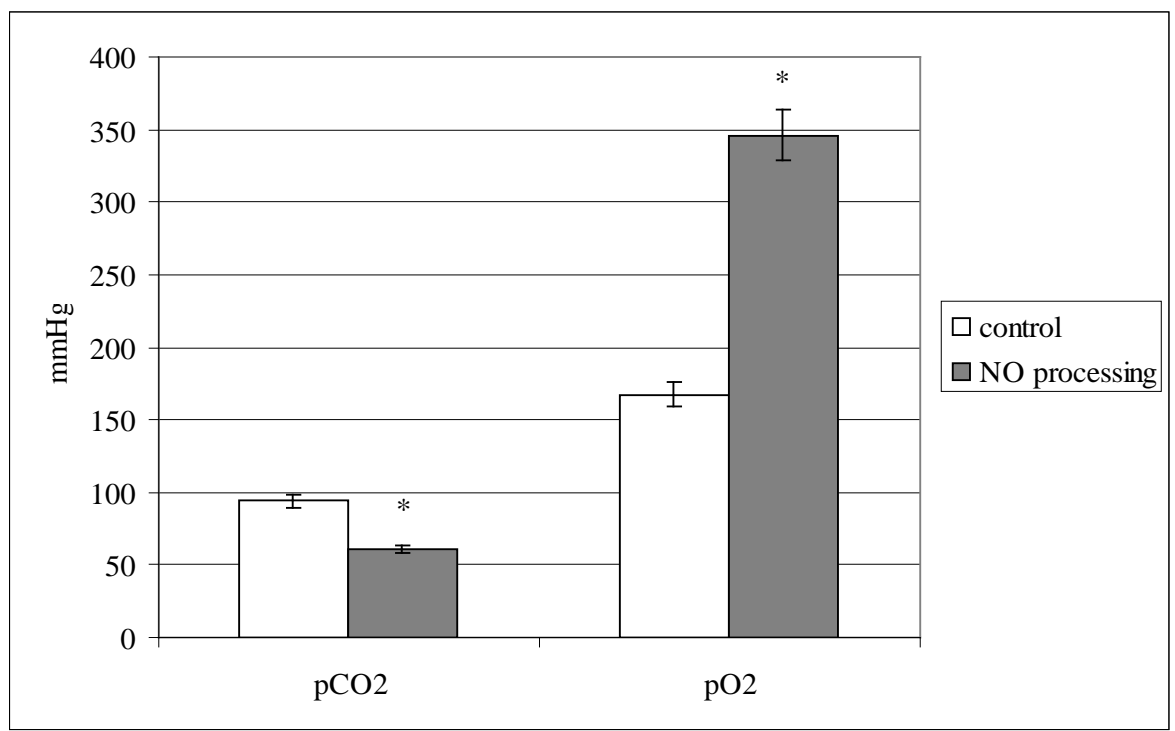

Figure 4. Partial pressure level of blood gases (in $\mathrm{mmHg}$ ) at gaseous nitric oxide processing ( $\mathrm{pCO}_{2}$ - partial pressure of the carbon dioxide; $\mathrm{pO}_{2}$ - partial pressure of the oxygen).

Before A. B. Shekhter et al. (2005) had showed, that skin wound processing by nitric oxide generating with "Plazon" apparatus had not any negative effects in vivo [6], observed at blood nitroxylation in vitro. On the contrary, this regimen of NO percutaneous action led to stimulation of wounds healing at $25 \%-30 \%$ to control. It was stated, that tissue NO level decreased in 5 - 10 min after short (1 min) wound processing with nitric oxide, and further its concentration was elevated. In 30 - 60 min after exposure NO concentration in wound tissue increased considerably to the initial level (at 10 - 20 times). We supposed primary reduction of NO level in wound associated with its interaction with superoxide anions and peroxynitrite accumulation with oxidative stress generation in exposed tissues. These processes can activate different antioxidant systems, which eliminate tissue superoxide abundance. It saved endogenic NO molecules, constantly producing with different species of NOsynthase, and it maked comfortable conditions for wound metabolism, determined its healing time and result. 
Thereby, stimulation of wound healing processes may be induced endogenic bioavalable, but not exogenic NO.

In this connection, stated complex reaction may be universal mechanism of human and animals organism response on sharp elevation of tissue NO level. Observed in our experiments negative NO effects on human blood specimens in vitro also may be caused by a low level of protective systems in isolated blood, which is incapable to NO-induced oxidative stress prevention.

\section{Conclusions}

Our experiments have showed that the whole human blood processing with gas flow NO-contained (NO concentration $800 \mathrm{mcg} / \mathrm{l}$ ) causes strong changes of its physical and chemical parameters. This exposure leads to inhibition of erythrocytes energy metabolism, decreasing plasma antioxidant reserves, moderate ionic disorders and of acid-base misbalance in blood samples in vitro. In addition, indirect signs indicate, that NO-processing in used regimen, mainly affecting erythrocytes, stipulates methemoglobin formation. These data testify that the used dose of gaseous nitric oxide is too high for human blood. In our opinion, registered negative effects of free NO may be eliminated by the bound nitric oxide use (first of all, its natural form-dinitrosyl-iron complexes (DNIC) [3] [4]). Really, at animal model intrawound injection (5 mcM) of DHIC with glutathione or cysteine led to increasing granulocytes number at 3 - 4 times to the control group without healing stimulation [31]. It can be occurred through DNIC possibility as interceptor of superoxide anions [32], that led to elevation of tissue NO concentration. In this case NO level increasing may happened by its releasing from DNIC and NO-synthases activity.

In addition, DNIC intravenous injections do not cause clear changes of blood homeostasis parameters at healthy volonteers [4]. Now character and mechanisms of its action on isolated blood samples are being investigated by us.

In addition, complex investigation of biological effects of free and bound nitric oxide in vitro and in vivo should be held for development of new medical technologies. For this task we can use the inhalation of gaseous nitric oxide or infusion of its bound form-DNIC. It can be useful for elimination of oxidative stress and optimization of metabolism in posttraumatic period.

\section{References}

[1] Gryglewsky, R.J. and Minuz P. (2001) Nitric Oxide. Basic Research and Clinical Application. IOS Press, Amsterdam, Berlin, Oxford, Tokyo, Washington.

[2] Murad, F. (1994) The Role of Nitric Oxide in Modulating Guanylyl Cyclase. Neurotransmissions, 10, 1-4.

[3] Vanin, A.F. (2009) Dinitrosyl-Iron Complexes with Thiolate Ligands: Physico-Chemistry, Biochemistry and Physiology. Nitric Oxide: Biology and Chemistry, 21, 136-149. http://dx.doi.org/10.1016/j.niox.2009.03.005

[4] Chazov, E.I., Rodnenkov, O.V., Zorin, A.V., et al. (2012) Hypotensive Effect of Oxacom Containing a Dinitrosyl Iron Complex with Glutathione. Animal Studies and Clinical Trials on Healthy Volunteers. Nitric Oxide: Biology and Chemistry, 26, 148-156. http://dx.doi.org/10.1016/j.niox.2012.01.008

[5] Van Faassen, E. and Vanin, A.F. (2007) Radicals for Life: The Various Forms of Nitric Oxide. Elsevier, Amsterdam.

[6] Shekhter, A.B., Serezhenkov, V.A., Rudenko, T.G., et al. (2005) Beneficial Effect of Gaseous Nitric Oxide on the Healing of Skin Wounds. Nitric Oxide: Biology and Chemistry, 12, 210-219. http://dx.doi.org/10.1016/j.niox.2005.03.004

[7] Martusevich, A.K., Peretyagin, S.P. and Ivannikova, E.V. (2012) Physical and Chemical Properties of Physiological Solution at Action of Oxygen and Nitrogen Reactive Species. Fundamental Research, 11, 197-201.

[8] Duarte, S., Kuo, S.P., Murata, R.M., et al. (2011) Air Plasma Effect on Dental Disinfection. Physics of Plasmas, 18, 073501-073507. http://dx.doi.org/10.1063/1.3606486

[9] Lai, W., Lai, H., Kuo, S.P., et al. (2005) Decontamination of Biological Warfare Agents by a Microwave Plasma Torch. Physics of Plasmas, 12, 023501-023506. http://dx.doi.org/10.1063/1.1843131

[10] Kuo, S.P. (2012) Air Plasma for Medical Applications. J. Biomedical Science and Engineering, 5, 481-495. http://dx.doi.org/10.4236/jbise.2012.59061

[11] Baxter, H.C., Campbell, G.A., Whittaker, A.G., et al. (2005) Elimination of TSE Infectivity and Decontamination of Surgical Instruments Using RF Gas-Plasma Treatment. Journal of General Virology, 86, 2393-2399. http://dx.doi.org/10.1099/vir.0.81016-0

[12] Kuo, S.P., Tarasenko, O., Popovic, S. and Levon, K. (2006) Killing of Bacterial Spores Contained in a Paper Envelope 
by a Microwave Plasma Torch. IEEE Transactions on Plasma Science, 34, 1275-1280. http://dx.doi.org/10.1109/TPS.2006.878389

[13] Kuo, S.P., Chen, C.Y., Lin, C.S. and Chiang, S.H. (2010) Wound Bleeding Control by Low Temperature Air Plasma. IEEE Transactions on Plasma Science, 38, 1908-1914. http://dx.doi.org/10.1109/TPS.2010.2047028

[14] Kuo, S.P., Chen, C.Y., Lin, C.S. and Chiang, S.H. (2012) Applications of Air Plasma for Wound Bleeding Control and Healing. IEEE Transactions on Plasma Science, 40, 1117-1123. http://dx.doi.org/10.1109/TPS.2012.2184142

[15] Brune, B. and Hanstein, K. (1998) Rapid Reversibility of Nitric Oxide Induced Platelet Inhibition. Thrombosis Research, 90, 83-91. http://dx.doi.org/10.1016/S0049-3848(98)00024-3

[16] Gries, A., Bode, C., Peter, K., et al. (1998) Inhaled Nitric Oxide Inhibits Human Platelet Aggregation, p-Selectin Expression, and Fibrinogen Binding in Vitro and in Vivo. Circulation, 97, 1481-1487. http://dx.doi.org/10.1161/01.CIR.97.15.1481

[17] Nong, Z., Hoylaerts, M., Van Pelt, N., et al. (1997) Nitric Oxide Inhalation Inhibits Platelet Aggregation and PlateletMediated Pulmonary Thrombosis in Rats. Circulation Research, 81, 865-869. http://dx.doi.org/10.1161/01.RES.81.5.865

[18] Weber, A., Strobach, H. and Schror, K. (1993) Direct Inhibition of Platelet Function by Organic Nitrates via Nitric Oxide Formation. European Journal of Pharmacology, 247, 29-37. http://dx.doi.org/10.1016/0922-4106(93)90134-U

[19] Giliano, N.Ya., Konevega, L.V., Noskin, L.A., et al. (2011) Dinitrosyl Iron Complexes with Thiol-Containing Ligands and Apoptosis: Studies with HeLa Cell Cultures. Nitric Oxide: Biology and Chemistry, 24, 151-159. http://dx.doi.org/10.1016/j.niox.2011.02.005

[20] Mathisen, D.J., Kuo, E.Y., Hahn, C., et al. (1998) Inhaled Nitric Oxide for Adult Respiratory Distress Syndrome after Pulmonary Resection. The Annals of Thoracic Surgery, 66, 1894-1902. http://dx.doi.org/10.1016/S0003-4975(98)01167-9

[21] Ricciardi, M.J., Knight, B.P., Martinez, F.J. and Rubenfire, M. (1998) Inhaled Nitric Oxide in Primary Hypertension: A Safe and Effective Agent for Predicting Response to Nifedipine. Journal of the American College of Cardiology, 32, 1068-1073. http://dx.doi.org/10.1016/S0735-1097(98)00361-1

[22] van der Vliet, A., et al. (1997) Formation of Reactive Nitrogen Species during Peroxidase-Catalyzed Oxidation of Nitrite. A Potential Additional Mechanism of Nitric Oxide-Dependent Toxicity. The Journal of Biological Chemistry, 272, 7617-7625. http://dx.doi.org/10.1074/jbc.272.12.7617

[23] Onufriev, M.V. (2010) Nitrosative Stress in the Brain: Autoantibodies to Nitrotyrosine in Liquor as Potential Marker, Neurochemistry, 27, 257-263.

[24] Martusevich, A.K. and Peretyagin, S.P. (2013) Modification of Blood Plasma Crystallogenesis with Nitrogen Oxide Processing. Biophysics, 58, 1038-1042. http://dx.doi.org/10.1134/S0006350913060134

[25] Hall, C.N. and Garthwaite, J. (2009) What Is the Real Physiological NO Concentration in Vivo? Nitric Oxide: Biology and Chemistry, 12, 92-103. http://dx.doi.org/10.1016/j.niox.2009.07.002

[26] Martusevich, A.K., Soloveva, A.G., Peretyagin, S.P. and Mitrofanov V.N. (2013) Estimation of Some Physical Factors Influence on Blood Energy Metabolism in Vitro. Biomedicine, 1, 103-108.

[27] Schweitzer, C. and Schmidt, R. (2003) Physical Mechanisms of Generation and Deactivation of Singlet Oxygen. Chemical Reviews, 103, 1685-1757.

[28] Genestra, M. (2007) Oxyl Radicals, Redox-Sensitive Signalling Cascades and Antioxidants. Cellular Signalling, 19, 1807-1819. http://dx.doi.org/10.1016/j.cellsig.2007.04.009

[29] Halliwell, B. and Gutteridge, J.M.C. (1999) Free Radicals in Biology and Medicine. Oxford University Press, Oxford.

[30] He, X., Azarov, I., Jeffers A., et al. (2008) The Potential of Angelis Salt to Decrease Nitric Oxide Scavenging by Plasma Hemoglobin. Free Radical Biology Medicine, 44, 1420-1432. http://dx.doi.org/10.1016/j.freeradbiomed.2007.12.038

[31] Shekhter, A.B., Rudenko, T.G., Serezhenkov, V.A. and Vanin, A.F. (2007) Dinitrosyl Iron Complexes with Thiol Ligands Promote Skin Wound Healing in Animals. Biophysics, 52, 539-547. http://dx.doi.org/10.1134/S0006350907050120

[32] Shumaev, K.B., Gubkin, A.A., Serezhenkov, V.A., et al. (2008) Interaction of Reactive Oxygen and Nitrogen Species with Albumin- and Methemoglobin-Bound Dinitrosyl Iron Complexes. Nitric Oxide: Biology and Chemistry, 18, 37-46. http://dx.doi.org/10.1016/j.niox.2007.09.085 
Scientific Research Publishing (SCIRP) is one of the largest Open Access journal publishers. It is currently publishing more than 200 open access, online, peer-reviewed journals covering a wide range of academic disciplines. SCIRP serves the worldwide academic communities and contributes to the progress and application of science with its publication.

Other selected journals from SCIRP are listed as below. Submit your manuscript to us via either submit@scirp.org or Online Submission Portal.
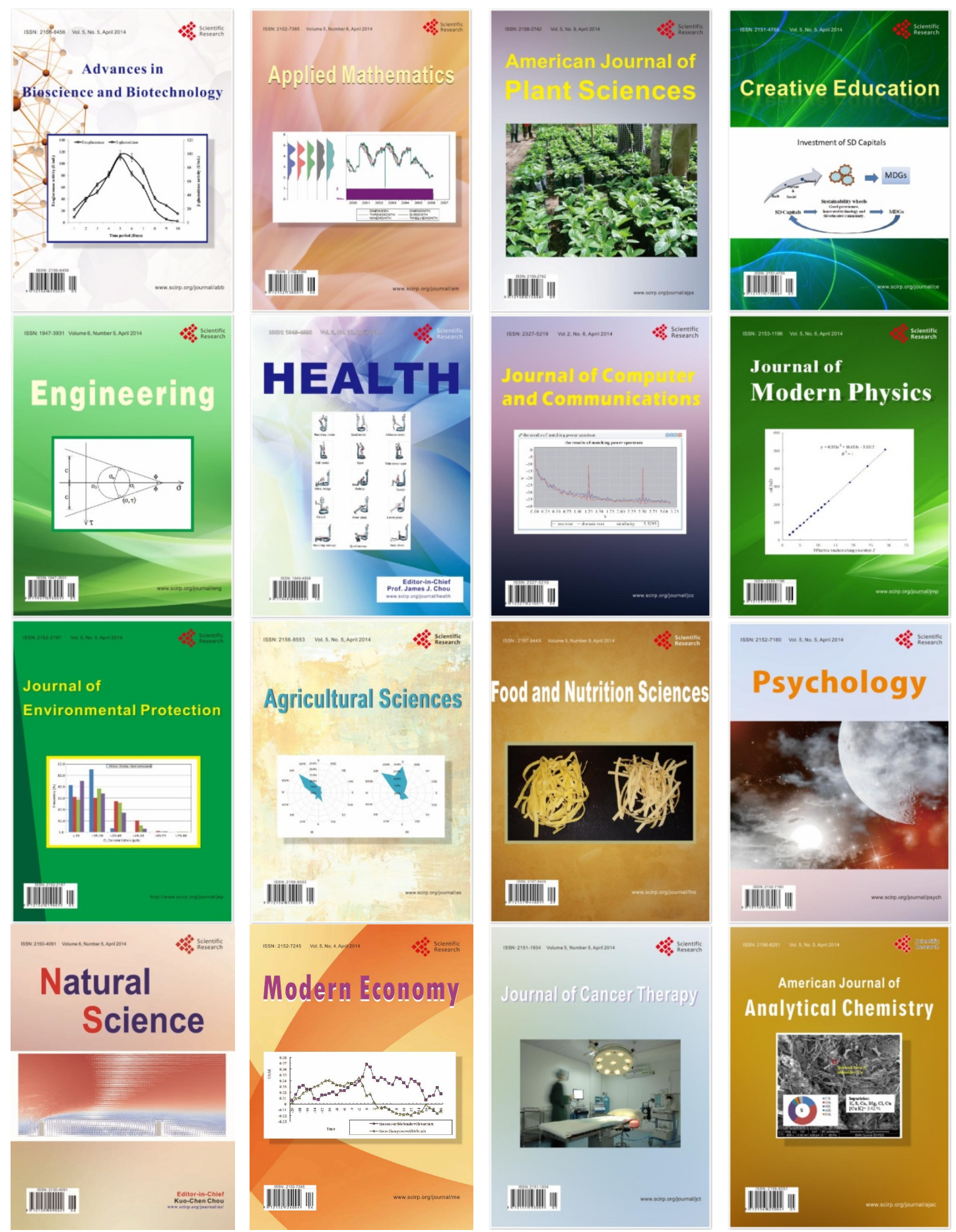